\title{
A Pair Production Telescope for Medium-Energy Gamma-Ray Polarimetry
}

\author{
Stanley D. Hunter ${ }^{1 \mathrm{a}}$, Peter F. Bloser ${ }^{\mathrm{b}}$, Gerardo O. Depaola ${ }^{\mathrm{c}}$, Michael P. Dion ${ }^{\mathrm{d}}$, \\ Georgia A. DeNolfo ${ }^{a}$, Andrei Hanu ${ }^{a}$, Marcos Iparraguirre ${ }^{c}$, Jason Legere ${ }^{b}$, Francesco Longo ${ }^{\text {e }}$, \\ Mark L. McConnell ${ }^{\text {b }}$, Suzanne F. Nowicki ${ }^{\text {a,f }}$, James M. Ryan ${ }^{\text {, }}$, \\ Seunghee Son ${ }^{\text {a,f }}$, and Floyd W. Stecker ${ }^{\text {a }}$ \\ ${ }^{a}$ NASA/Goddard Space Flight Center, Greenbelt Road, Greenbelt, MD 20771 \\ ${ }^{\mathrm{b}}$ Space Science Center, University of New Hampshire, Durham, NH 03824 \\ ${ }^{\mathrm{c}}$ Facultad de Matemática, Astronomía y Fisica, Universidad de Córdoba, \\ Córdoba 5008 Argentina \\ ${ }^{d}$ Pacific Northwest National Laboratory, Richland, WA 99352 \\ ${ }^{\mathrm{e}}$ Dipartimento di fisica, Università Degli Studi de Trieste, Treste Italy \\ ${ }^{\mathrm{f}}$ Department of Physics, University of Maryland Baltimore County, Baltimore, MD 21250
}

\begin{abstract}
We describe the science motivation and development of a pair production telescope for mediumenergy ( 5-200 MeV) gamma-ray polarimetry. Our instrument concept, the Advanced Energetic Pair Telescope (AdEPT), takes advantage of the Three-Dimensional Track Imager, a low-density gaseous time projection chamber, to achieve angular resolution within a factor of two of the pair production kinematics limit $\left(\sim 0.6^{\circ}\right.$ at $\left.70 \mathrm{MeV}\right)$, continuum sensitivity comparable with the Fermi-LAT front detector $\left(<3 \times 10^{-6} \mathrm{MeV} \mathrm{cm}^{-2} \mathrm{~s}^{-1}\right.$ at $\left.70 \mathrm{MeV}\right)$, and minimum detectable polarization less than $10 \%$ for a $10 \mathrm{mCrab}$ source in $10^{6}$ seconds.
\end{abstract}

Keywords: gamma rays, telescope, pair production, angular resolution, polarimetry, sensitivity, time projection chamber, astrophysics

${ }^{1}$ Corresponding author at: NASA/GSFC, Code 661, Greenbelt, MD 20771, USA. Tel: +1 301 286-7280; fax +1 301 286-0677, E-mail address: stanley.d.hunter@nasa.gov phone; 


\section{Introduction}

Since the launch of AGILE [1] and Fermi/LAT [2], the scientific progress in high-energy $\left(E_{\gamma} \gtrsim 200 \mathrm{MeV}\right.$ ) gamma-ray science has been extensive. Both of these telescopes cover a broad energy range from $\sim 20 \mathrm{MeV}$ to $>10 \mathrm{GeV}$. However, neither instrument is optimized for observations below $200 \mathrm{MeV}$ or for polarization sensitivity. Ground-based air Cherenkov telescopes have been used to observe both galactic sources such as supernova remnants and extragalactic sources of very high energy (TeV) gamma-rays such as active galactic nuclei (AGN) [3]. They have provided important astrophysical information, but they also lack the capability to detect polarization. The Fermi and AGILE space-based telescopes, operating in the $\mathrm{GeV}$ energy range, are expected to continue to make significant progress for the next several years. However, there remains a significant gap in our knowledge of astronomy in the mediumenergy ( 0.1-200 MeV) regime between the X-ray and high-energy gamma-ray energy ranges.

The next major step in gamma-ray astrophysics, recognized as early as the SAS-2 era [4], should be a medium-energy gamma-ray pair production telescope to fill this gap and provide answers to many important astrophysical questions. In the following, we describe the science motivation for this mission and the design of the Advanced Energetic Pair Telescope (AdEPT) a pair production telescope for medium-energy, $\sim 5$ to $\sim 200 \mathrm{MeV}$, gamma-ray polarimetry.

\section{Science Motivation}

The AdEPT pair production telescope for the detection of medium energy $(\sim 5-200 \mathrm{MeV})$ gamma-rays with high angular resolution and polarimetry capabilities will open a new window in observational astronomy and astrophysics. Such an instrument can help provide answers to important questions in both astronomy and physics. For example, it can shed light on the origin and acceleration of cosmic rays, the nature of the cosmic-ray acceleration of electrons in the Crab nebula to energies in excess of $10^{15}$ electron volts [5] and how pulsars, with high magnetic fields and expected high gamma-ray polarization, achieve such high efficiency for particle acceleration. Gamma-ray polarization can distinguish between emission processes such as synchrotron radiation and other gamma-ray production mechanisms, however, the angular resolution with which the geometry of the gamma-ray emission regions are probed by polarization measurements is limited by the instrument angular resolution. It has long been 
expected that other astronomical sources such as "blazars" (a class of active galactic nuclei) should produce polarized gamma-radiation owing to the highly structured magnetic fields in their emission regions [6][7][8][9]. It is also known that gamma-ray bursts (GRBs) emit hard Xradiation whose polarization has been detected by space borne instruments, e.g. RHESSI [10], INTEGRAL [11], and GAP[12]. Such polarization should extend into the gamma-ray range, given the same basic emission processes. Observations at higher energies will investigate an underexplored energy range and provide new understanding of emission mechanisms with high polarization sensitivity.

Medium energy polarization measurements with AdEPT can also explore fundamental questions in theoretical physics. There is an apparent incompatibility between relativity and quantum mechanics at the Planck scale of $1.6 \times 10^{-35}$ meters. Effective field theory models developed to determine possible quantum gravity effects at observable energies, have led to the prediction of possible "vacuum birefringence", a process in which photons of different polarizations travel at slightly different velocities from an astronomical source. Such a process, if it exists at a significant enough scale, can destroy the inherent polarization of a source from which such polarization would be seen in its absence. Thus, the detection of polarization from a distant source such as a gamma-ray burst can constrain the possible existence of violations of relativity [13][14]. The birefringence effect is sensitive to the square of the photon energy. To date, the INTEGRAL/IBIS observations of the Crab pulsar and nebula at 200-800 keV [15] are the highest energy photon polarization measurements that have been made. An instrument capable of detecting polarization of medium energy gamma-rays can provide a much more sensitive probe of such relativity violations.

The AdEPT pair production telescope also has significant advantages over previous attempts to measure the medium-energy diffuse extragalactic gamma-ray background. Possible contributing components [16] include non-thermal tails from Seyfert galaxies, red-shifted lines from Type Ia and Type II supernovae, and unknown extragalactic sources. Measurements by both the Apollo21 [17] and COMPTEL [18] instruments were plagued by intrinsic detector and spacecraft background problems owing to the buildup of long-lived radioisotopes created by cosmic-ray interactions. The subtraction of such poorly determined backgrounds led to uncertainties in the extragalactic background determination and significantly different results reported by the two different instruments. A free-flying argon gas AdEPT instrument is expected to have low 
intrinsic background similar to EGRET and Fermi/LAT and therefore yield a more reliable determination of the extragalactic gamma-ray background in the medium-energy range.

The 5-plus fold improvement in angular resolution of AdEPT below 200 MeV compared with Fermi/LAT, see Figure 1 will enable the numerous soft gamma-ray sources in the galactic plane to be better resolved improving the determination of the medium-energy Galactic diffuse emission and to spatially resolve variation between electron dominated and hadron dominated processes in the 70-200 MeV range.

\section{Obtainable Goals for Exploring the Medium Energy Gamma-ray Universe}

Significant improvement in sensitivity for pair telescopes can only be achieved through a dramatic improvement in the angular resolution, especially at lower energies. The ultimate angular resolution of any nuclear pair-production telescope is limited by the unobserved recoil momentum of the nucleus. The nuclear recoil momentum calculated by Jost, Luttinger \& Slotnick [19] for photon energy $E_{\gamma}$ has a broad distribution extending from $2 \mathrm{~m}_{\mathrm{e}}^{2} / E_{\gamma}$ to $E_{\gamma}$, where $\mathrm{m}_{\mathrm{e}}$ is the electron rest mass, and the nuclear momentum is nearly orthogonal to the gamma-ray momentum. On the assumption that the recoil momentum is transverse to the photon direction [20], an upper limit to the kinematic limit can be defined as $q_{68} / E_{\gamma}$, where $q_{68}$ is the momentum above which $68 \%$ of the distribution lies. This assumption is not valid at energies below $\sim 25 \mathrm{MeV}$ where the momentum distribution is wider and the recoil angle is more acute. The kinematic limit and twice the limit are shown in Figure 1 as the solid and dotted magenta lines, respectively. In the case of triplet production, i.e. pair production on the atomic electrons, the recoil momentum is, in most cases, observable [21] and the angular resolution is limited by the energy and spatial resolution of the electron track imager. Further discussion of triplet detection with AdEPT including effective area (enhanced for low-Z materials), angular resolution, and polarization asymmetry factor is beyond the scope of this paper and will be addressed in a future paper.

The performance goals of a telescope to address the questions outlined above plus a wide variety of other interesting topics including solar flares, diffuse emission, etc. are summarized in Table 1.

Table 1 - AdEPT Instrument Performance Goals 


\begin{tabular}{rl|}
\hline Energy range & $\sim 5$ to $\sim 200 \mathrm{MeV}$ \\
Energy resolution & $\sim 30 \% \Delta \mathrm{E} / \mathrm{E}(\mathrm{FWHM}$ ) at $\sim 70 \mathrm{MeV}$ \\
Angular resolution & $<1^{\circ}$ at $70 \mathrm{MeV}$ \\
Continuum sensitivity $\times \mathrm{E}^{2}$ & $\sim 3 \times 10^{-5} \mathrm{MeV} \mathrm{cm}^{-2} \mathrm{~s}^{-1}$ \\
Minimum detectable polarization & $\sim 10 \%$ for $10 \mathrm{mCrab}^{\circ}$ in $10^{6} \mathrm{~s}$ \\
& (asymmetry factor, $\lambda=0.15)$
\end{tabular}

In the following, we show that a medium energy gamma-ray telescope meeting these angular resolution and polarization sensitivity requirements can be developed and describe the design of the Advanced Energetic Pair Telescope (AdEPT) ${ }^{2}$ the telescope presently being developed using the Three-Dimensional Track Imager (3-DTI) technology developed at Goddard Space Flight Center (GSFC). In the following sections we describe the advantages of the 3-DTI gas detector technology and the predicted performance of AdEPT.

\section{Advantages of a Gas Detector for a Pair Production Telescope}

The design of all pair production space telescopes to date, SAS-2 [22], COS-B [23], EGRET [24], AGILE [1], and Fermi-LAT [2] have utilized an electron tracking hodoscope consisting of a stack of electron tracking detectors interleaved with metal foils, each typically 20 milliradiation lengths (mRL) thick, positioned above a calorimeter. SAS-2, COS-B and EGRET utilized two-dimensional gas spark chambers whereas AGILE and Fermi/LAT have taken advantage of silicon-strip detectors (SSD). The multiple layers of high-Z metal foils (totaling about $500 \mathrm{mRL}$ ) provide substantial material for high interaction probability and large effective area, however, they also contribute to multiple Coulomb scattering (MCS) which degrades the accuracy with which the electron and positron directions emanating from the pair vertex can be determined. Kryshkin, Sterligov, \& Usov [25] determined that these directions for high energy $(900 \mathrm{MeV})$ gamma rays, which form the basis of gamma-ray direction and polarization determination, are dominated by MCS after traversing about $10 \mathrm{mRL}$ of material. The maximum material thickness would be even less for lower energy gamma rays.

In low-Z material, $Z \lesssim 30$, gamma rays with energy below $\sim 10 \mathrm{MeV}$, are more likely to interact via Compton scattering than pair production, however, the intrinsic modulation factor of polarized gamma rays interacting via pair production is higher above $\sim 2 \mathrm{MeV}$, compared to Compton scattering and photo-electric absorption [26]. Thus, we are motivated to reduce the

\footnotetext{
${ }^{2}$ A list of less common acronyms is given at the end this paper.
} 
effective minimum energy of a pair telescope towards the threshold energy, to take advantage of the higher modulation factor. This requires that the direction of the electron and positron emanating from the pair vertex, which forms the basis of the gamma-ray direction and polarization determination, be measured in less than $\sim 10 \mathrm{mRL}$ of material after which their directions are dominated by multiple Coulomb scattering (MCS) [25].

In the remainder of this section we give a detailed calculation that corroborates the conclusion that a low density, less than $\sim 5 \mathrm{mg} / \mathrm{cm}^{3}$, track imager is required to achieve the AdEPT performance.

\subsection{Electron track measurement constraint}

Achieving high angular resolution and the lowest possible minimum detectable polarization (MDP) requires a new approach to reduce the density of the conversion and scattering material per track measurement interval in the hodoscope. The density per measurement interval (measurement density) of a hodoscope with interleaved foils can be reduced by decreasing the thickness of the conversion material or increasing the separation between the measurement layers.

The concept of reducing the thickness of the converter material in a gamma-ray telescope to improve the medium-energy sensitivity was recognized by Kniffen, et al. [27]. They achieved nearly an order of magnitude increase in sensitivity at $20 \mathrm{MeV}$ by replacing the lead conversion foils in a gas spark-chamber telescope, used previously for high-energy gamma-ray observation [28], with aluminum foils. More recently, several pair telescopes have been proposed without conversion foils, i.e. the conversion material is the SSDs of the track imager. Proposed applications of this concept are MEGA [29], TIGRE [30], and a GLAST/LAT modification [31].

The AdEPT gamma-ray telescope concept (Table 3) takes advantage of a gaseous medium to provide a homogenous tracking detector to achieve nearly continuous measurements of the electron and positron tracks from pair production. The optimal fit formulas derived by Innes [32] for estimating the tracing parameter error matrix in the case of a homogenous detector with many layers, can be used to estimate the AdEPT angular resolution and place an upper limit on the gamma-ray convertor density.

Innes describes the projection of a nearly straight track onto the plane perpendicular to the 
magnetic field as $y=a+b x+c x^{2} / 2$ where $a$ is the position at the beginning of the track, $b$ is the slope, $c$ is the curvature, and $x$ is the distance along the track. Innes defines the information density of a detector with total length $L, N$ equally spaced measurement layers, and RMS measurement error at each layer $\sigma_{\mathrm{m}}$ as $\iota=(N+5) / \sigma_{\mathrm{m}}^{2} L$. The information density of the EGRET [33], MEGA [29], Fermi-LAT front section (hereafter Fermi/LAT) [34], and AGILE [35] telescopes along with the AdEPT concept (Table 3) are listed in Table 2. We have restricted the AdEPT detector length, corresponding to selection of the initial portion of the electron track, and taken an upper limit to the measurement spacing, see §5.2.

Table 2 - Gamma-ray Telescope Information Density

\begin{tabular}{|c|c|c|c|c|c|}
\hline Telescope & $N$ & $L(\mathrm{~mm})$ & $l=L / N(\mathrm{~mm})$ & $\begin{array}{c}\text { Pitch }(\mathrm{mm}) \\
\sigma_{\mathrm{m}}=P / \sqrt{12}\end{array}$ & $\iota\left(\mathrm{mm}^{-3}\right)$ \\
\hline EGRET & 28 & 450 & 16.1 & 0.810 & 1.33 \\
\hline MEGA & 32 & 320 & 10.0 & 0.470 & 6.28 \\
\hline Fermi/LAT & 12 & 416 & 34.7 & 0.228 & 9.42 \\
\hline AGILE & 12 & 228 & 19.0 & 0.242 & 17.38 \\
\hline AdEPT & 300 & 300 & 1.0 & 0.400 & 76.25 \\
\hline
\end{tabular}

Innes further defines the characteristic scattering length $\ell=1 / \sqrt[4]{\iota s}$ in terms of the information density and $s$ the mean square projected scattering angle per unit length. Omitting the small logarithmic term in the Gaussian approximation for the central $98 \%$ of the projected scattering angular distribution [36][37], we have $s \approx(13.6 \mathrm{MeV} / \beta c p)^{2} \cdot 1 / X_{0}^{\prime}$, where $X_{0}^{\prime}=X_{0} / \rho$ is the $\mathrm{RL} / \mathrm{density}$ in units of mm. Innes derives formulas (Eq. 11 of [32]) for the optimal fit variance matrix elements in the continuous detector limit $(l \rightarrow 0$, with $\iota$ and $s$ constant, for large $N)$ in the limiting case, $u=L / \ell>7$ where the tracking error is dominated by MCS, and $u<7$, where the error is determined by the tracer spatial resolution. For the AdEPT concept described in Tables 2 $\& 3, u$ is well approximated by $u \approx 60.9 \cdot \sqrt{10 \mathrm{MeV} / E_{e}}$ for electron energies above $\sim 0.5 \mathrm{MeV}$. Over the medium-energy range, $u \gg 7$ and the AdEPT angular resolution is dominated by MCS and we do not consider the correction to the Innes equations for $u<7$ in the absence of a magnetic field noted by Bernard [38]. For nearly straight tracks in the absence of magnetic field curvature, the variance of the optimum fit track slope is given by the $V_{b b}$ matrix element 


$$
\sigma_{\theta t}^{2}=V_{b b} \approx 1 /{ }_{\iota} \ell^{3}(2 / u+\sqrt{2}) \stackrel{u \gg 1}{\longrightarrow} \sqrt{2} / /_{\imath \ell^{3}}
$$

We note that this formula is not applicable to MEGA, EGRET, Fermi/LAT, and AGILE because of their small number of measurement layers and discrete convertor foils.

The gamma-ray direction, in the small-angle approximation, is reconstructed by combining the measured directions of the electron and positron weighted by their energies (see $\$ 3.11$ of [20]). In the multiple scattering dominated regime, the conversion of track resolution to photon angular resolution is $\sigma_{\theta_{\gamma}}=\sigma_{\theta_{t}} \sqrt{\sqrt{r}+\sqrt{1-r}}$ where $r$ and $1-r$ are the energy fractions carried away by the electron and positron. In the case of equal energy partition $(r=0.5)$ we have $\sigma_{\theta_{\gamma}} \approx 1.2 \sigma_{\theta_{t}}$, which becomes $\sigma_{\theta_{\gamma}}=\sigma_{\theta_{t}}$, for asymmetric energy partition, $r \approx 0$.

The point spread function of a gamma-ray telescope is often expressed in terms of the $68 \%$ containment angle $\theta_{68}$, which is related to the standard deviation of a Gaussian distribution by

$$
\theta_{68}=\sqrt{-2 \cdot \ln (1-0.68)} \sigma_{\theta \gamma} \approx 1.51 \sigma_{\theta \gamma} .
$$

The expected angular resolution of the AdEPT telescope is the quadrature sum of the kinematic limit and $\theta_{68}$ and is shown as the solid black line in Figure 1. Up to some energy the angular resolution is dominated by the kinematic limit. We denote the gamma-ray energy at which the angular resolution is twice the kinematic limit, indicated by the vertical black dotted line in Figure 1 , as $E_{\gamma, \mathrm{KL}} \approx 150 \mathrm{MeV}$. The corresponding angular resolution, $\theta_{68, \mathrm{KL}} \approx 6 \mathrm{mrad}$, indicated by the horizontal black dotted line determines the electron-positron direction error $\sigma_{\theta t, \mathrm{KL}} \approx 3.36 \mathrm{mrad}$ and, in the limit $u \gg 1$, Eq. (1) can be used to estimate the scattering angle, $s$, and hence the RL of the electron-positron tracking medium.

$$
S=\sqrt[3]{\sigma_{\theta t}^{8} \iota / 4} \approx\left(\frac{13.6}{\beta c p}\right)^{2} \frac{1}{X_{0}^{\prime}}
$$

Using the $\theta_{68}$ value from Figure 1 and assuming $E_{e}=E_{\gamma} / 2-\mathrm{m}_{e}$ the value of $X_{0}^{\prime}$ increases from $\sim 0.15 \times 10^{4} \mathrm{~cm}$ at $5 \mathrm{MeV}$ to $\sim 1.75 \times 10^{4} \mathrm{~cm}$ at $\sim 800 \mathrm{MeV}$. On the assumptions, $\mathrm{A} \approx 2.1 \mathrm{Z}$ for low-Z materials $(\mathrm{Z}<20), \mathrm{A} \approx 2.5 \mathrm{Z}-8$ for higher $\mathrm{Z}$ materials $(20<Z<54)$, and using Dahl's approximation [37] for the RL of a material, $X_{0}=\frac{716.4 \mathrm{~A}}{\mathrm{Z}(\mathrm{Z}+1) \ln (287 / \sqrt{\mathrm{Z}})} \mathrm{g} / \mathrm{cm}^{2}$, the density of 
the tacking material must lie between $\sim 0.5$ and $\sim 5 \mathrm{mg} / \mathrm{cm}^{3}$. With the exception of modern aerogel materials [39] this low density can only be realized with a gaseous media.

\subsection{Polarization constraint}

The use of the azimuthal orientation of the electron positron plane to determine the gamma ray polarization has been discussed since the 1950s [40][41] and imposes another constraint on the track imager. In the case of small nuclear recoil, i.e. the electron, positron, and incident photon are nearly coplanar, the azimuthal dependence of the cross section can be written in the form [42]

$$
\sigma(\phi)=\frac{\sigma_{0}}{2 \pi}\left[1+P \lambda \cos ^{2} \phi\right]
$$

where $\sigma_{0}$ is the total cross section, $\phi$ is the angle between the electron-positron pair and the photon's electric field vector, $P$ is the fractional polarization, and $\lambda$ is the inherent azimuthal asymmetry factor. Kel'ner, Kotov, and Logunov [26] calculated the azimuthal asymmetry of the secondary emission for the photoelectric, Compton, and pair production processes. They found that the asymmetry factor $\lambda$ is higher for photoelectric and pair production processes below and above $\sim 2 \mathrm{MeV}$ respectively compared to the Compton process and they showed that the polarization modulation factor and hence the polarization sensitivity, decreases exponentially with the thickness of material traversed by the pair electrons after only a few mRL [26]. These calculations assume that the electron and positron energies are greater than $1.5 \mathrm{MeV}$ and that the angle between them and the photon is less than $40^{\circ}$. These calculations support the conclusion of Mattox [43][44] that the thickness of the conversion foils in previous telescopes (typically $20 \mathrm{mRL}$ ) precludes any polarization sensitivity for these instruments. Buehler et al. [45] estimated that Fermi LAT might have marginal polarization sensitivity by selecting 50-200 MeV photons that convert in the silicon rather than the $30 \mathrm{mRL}$ thick tungsten foils. Their analysis, omitting background and trial factor considerations, concluded that $20 \%$ polarization from Vela could be detected at $3 \sigma$ using 20 months of data. The $8 \mathrm{mRL}$ thickness of the two silicon-strip detectors reduces the polarization sensitivity.

\subsection{Gaseous track imager}

Significant advances in medium-energy gamma-ray pair production telescopes can only be realized if the density of the material in the track imager is drastically reduced. A low-density, 
homogenous detector that provides high spatial resolution tracking and substantially minimizes the effects of Coulomb scattering is required. These technical challenges were met historically with a whole genre of detectors based on gas physics including cloud chambers, bubble chambers, and gas-wire detectors (spark and drift chambers, etc., see e.g. [46]). The use of a gaseous medium time projection chamber (TPC) as both the conversion and detection medium for a gamma ray pair telescope was first suggested by Hartman [47] and further explored by Bloser, et al. [48][49]; Hunter, et al. [50][51]; Ueno, et al. [52], and Bernard [20]. In the following section we describe our Three-Dimensional Track Imager (3-DTI), a gaseous time projection chamber technology, its application to the design of AdEPT, and the expected performance of the AdEPT pair polarimeter instrument.

\section{Three-Dimensional Track Imager (3-DTI)}

The 3-DTI detector, shown schematically in Figure 2, combines a gas Time Projection Chamber (TPC) [53][54] and a 2-D readout to provide a low density gamma-ray conversion medium with high-resolution, 3-D charged particle tracking obtained by digitizing the 2-D readout signals. The 3-DTI also takes advantage of Negative-Ion drift [55] to reduce diffusion to the thermal limit without an applied magnetic field allowing the TPC drift distance to be much larger than would be possible with free electron diffusion.

The TPC volume, which defines the 3-DTI active volume, is bounded by a drift electrode on the top, a linear potential gradient field-shaping cage of wires, and 2-D readout plane on the bottom. A charged particle traversing the gas medium loses energy by ionization. The ionization electron density is proportional to the $\mathrm{d} E / \mathrm{d} x$ energy loss of the particle along its track. The drift field, the electric field in the TPC active volume $(\sim 1 \mathrm{kV} / \mathrm{cm})$, causes the ionization to drift at a uniform velocity onto the 2-D readout plane. The relative 3-D location of the ionization charge is determined from the 2-D readout and time of arrival.

\subsection{Two-Dimensional Readout}

The 2-D readout consists of a 2-D Micro-Well Detector (MWD) [56][57][58][59][60] with preamplification provided by a Gas Electron Multiplier (GEM) [61]. Two-stage amplification of the ionization charge was required to detect single ionization electrons using the negative ion drift technique (see $\S 5.2,5.3$ ). The MWD consists of two orthogonal layers of electrodes separated by an insulating substrate, see lower right inset in Figure 2. The cathode and anode electrode 
strips are etched onto the top and bottom layers respectively of a copper clad insulator using flex circuit board technology. Holes etched in the top (cathode) electrodes are concentric with blind vias micro-machined in the insulator that expose the anode electrode and form the micro-wells. Our MWD design has $200 \mu \mathrm{m}$ diameter $\times 200 \mu \mathrm{m}$ deep wells on $400 \mu \mathrm{m} \times 400 \mu \mathrm{m}$ center-tocenter pitch. Charge entering a micro-well is accelerated by the strong electric field $(\sim 40 \mathrm{kV} / \mathrm{cm})$ in the wells and can produce a Townsend electron avalanche (e.g. [62]) proportional to the primary ionization charge. This amplification, or "gas gain", is exponentially dependent on the electric field in the micro-well. The avalanche electrons are collected on the anode and the motion of the avalanche charge induces an equal but opposite image charge on the cathode. The anode and cathode signals provide the 2-D, (X-Y), spatial location of the primary ionization. Sampling of the avalanche charge signal at a fixed frequency allows the third dimension $\mathrm{Z}$ (height) to be calculated from the uniform drift velocity of the ionization charge through the gas volume.

\subsection{Negative Ion Diffusion}

Diffusion of the ionization electrons drifting through the gas places an upper limit on the useful height of the TPC. If the maximum allowable diffusion is chosen to be twice the TPC readout pitch, then the maximum drift distance can be expressed in terms of the diffusion coefficient: $Z_{\max }=\left(2 \mathrm{Pitch} / \sigma_{0}\right)^{2}$. For example, Puiz [63] measured the electron drift velocity and diffusion in an $\mathrm{Ar}+\mathrm{CO}_{2}(80 \% / 20 \%)$ mixture at $1 \mathrm{~atm}$. The drift velocity of free electrons $V_{d}$ increases quasi-linearly with the drift field, $E$, with reduced mobility $\mu=V_{d} / E \approx 4.2 \times$ $10^{3} \mathrm{~cm}^{2} \mathrm{~atm} /$ Vs (Fig. 17 of [63]). The electron diffusion coefficient, $\sigma_{0}$, shown as the red line in

Figure 3, exhibits thermal behavior decreasing as $1 / \sqrt{2 k T / e E}$, blue line, up to $\sim 100 \mathrm{~V} / \mathrm{cm}$. For higher drift fields, the electron drift velocity is significantly higher than the thermal velocity of the gas and $\sigma_{0}$ tends to increase with $E$ reaching a plateau at high fields $\gtrsim 800 \mathrm{~V} / \mathrm{cm}$ atm. The minimum diffusion value of $\sigma_{0} \approx 180 \mu \mathrm{m} / \sqrt{\mathrm{cm}}$, is reached at $\sim 300 \mathrm{~V} / \mathrm{cm}$, corresponding to a drift velocity $V_{d}=\mu E \approx 1.2 \times 10^{4} \mathrm{~mm} / \mathrm{ms}$. The maximum drift distance in $\mathrm{Ar}+\mathrm{CO}_{2}$ is then $\sim 20 \mathrm{~cm}$ for a detector pitch of $400 \mu \mathrm{m}$.

Thermal diffusion can be maintained at higher fields by adding an electronegative component to the gas that captures the primary ionization electrons, forming negative ions, which then drift in thermal equilibrium with the gas. Carbon disulphide $\left(\mathrm{CS}_{2}\right)$, with a vapor pressure of $\sim 300$ torr at 
$300 \mathrm{~K}$ and moderate electron affinity [64], has successfully been used as a negative ion molecule in the Negative Ion-Time Projection Chamber (NI-TPC) [55]. We have measured the negative ion diffusion coefficient in $\mathrm{CH}_{4}+\mathrm{CS}_{2}$ [65] and in $\mathrm{Xe}$ at 100, 200, 300 torr plus 40 torr $\mathrm{CS}_{2}$. The Xe results are shown in Figure 3. For both gas mixtures, the negative ion diffusion coefficient is reduced to the thermal limit and is a function only of the drift field and gas temperature, and independent of the gas mixture. For negative ions, the diffusion coefficient decreases with $E$ becoming less than $\sim 80 \mu \mathrm{m} / \sqrt{\mathrm{cm}}$ above $\sim 800 \mathrm{~V} / \mathrm{cm}$ at $25^{\circ} \mathrm{C}$. In this case, the maximum drift distance for a detector pitch of $400 \mu \mathrm{m}$ is greater than $\sim 100 \mathrm{~cm}$. We note that the diffusion can be further reduced by operating the TPC at lower temperature, e.g. $0^{\circ} \mathrm{C}$ or lower.

The negative-ion drift technique allows for large TPC active volumes that can be read out with one readout layer, however, the drift velocity is substantially lower than for free electrons and, for constant voltage, the gas gain with $\mathrm{CS}_{2}$ added is reduced about 100-fold. The drift velocity of the $\mathrm{CS}_{2}^{-}$ions in $\mathrm{Xe}$ is $\sim 10 \mathrm{~mm} / \mathrm{ms}$ at $800 \mathrm{~V} / \mathrm{cm}$; about 3 orders of magnitude slower than that of free electrons in $\mathrm{Ar}+\mathrm{CO}_{2}$ [63]. Reduced drift velocity is an advantage in the digitization rate corresponding to a $\mathrm{z}$-coordinate resolution is reduced and the sampling rate of the digitizers can also be reduced which results in lower instrument power, see $\S 6 . \mathrm{CS}_{2}$ also provides strong UV quenching, which reduces breakdown brought on by the electron avalanche and ensures stable operation. The threshold for electron dissociation of $\mathrm{CS}_{2}$ is $9.337 \pm 0.06 \mathrm{eV}$ [66], thus, dissociation represents a negligible effect and gas degradation should be minimal ensuring a long instrument life-time.

Since thermal diffusion is independent of the gas mixture, we choose $\mathrm{Ar}+\mathrm{CS}_{2}$ rather than $\mathrm{Xe}+\mathrm{CS}_{2}$ for 3-DTI because of the higher drift velocity, reduced Coulomb scattering, and higher relative triplet production. Our measured mobility in $\mathrm{Ar}+\mathrm{CS}_{2}$ at 660 torr and $1200-1500 \mathrm{~V} / \mathrm{cm}$ is 16-20 mm/ms, consistent with the results of Ohnuki et al. [67].

\subsection{Single Ionization Electron Detection}

Detection of the ionization electrons along the tracks of the electron and positron pair is a requirement for gamma-ray imaging. Generation of a Townsend avalanche in the MWD requires detachment of the ionization electrons from the negative ions. This occurs in a strong electric field of the micro-well by collision of the negative ion with the gas molecules [68]. The free electrons are then accelerated in the micro-well producing the avalanche. The start of the 
avalanche from a detached electrons, however, occurs lower in the micro-well (closer to the anode) compared with a free electron avalanche resulting in lower gain for given MWD voltages, shown in Figure 4. The maximum electric field, and hence maximum avalanche gain, is determined by the maximum stable operating voltage of the micro-well detector corresponding with Raether's criterion in micro-pattern gas detectors. This was demonstrated by Ivaniouchenkov et al. [69] and Bressan et al. [70]. The micro-well voltage cannot be increased sufficiently to overcome the reduction in gain caused by the negative ion collision effect. We have overcome the reduction in gain by adding a Gas Electron Multiplier (GEM) [61] preamplification stage to our micro-well detector. The gain of our MWD with and without the GEM pre-amplifier measured in P-10 $\left(90 \% \mathrm{Ar}+10 \% \mathrm{CH}_{4}\right)$ and $\mathrm{Ar}+\mathrm{CS}_{2}(560$ torr +40 torr) at a total pressure of 600 torr is shown in Figure 4. Gains in excess of $10^{4}$ are readily achievable with the MWD+GEM combination in $\mathrm{Ar}+\mathrm{CS}_{2}$ providing single ionization electron detection. The $\mathrm{X}-$ $\mathrm{Z}$ projection of the electron-positron tracks resulting from the pair interaction of a $6.129 \mathrm{MeV}$ gamma ray is shown in Figure 5 obtained with a small $5 \times 5 \times 9 \mathrm{~cm}^{3}$ 3-DTI prototype with MWD+GEM readout. These highly structured tracks show pulse amplitude variation proportional to the $\mathrm{d} E / \mathrm{d} x$ energy loss of the electrons along their paths, multiple Coulomb scattering, and the formation of the Bragg peak of the stopping lower energy particle. X-Z and Y-Z projections of typical electron track from ${ }^{90} \mathrm{Sr}$ are shown in Figure 6. The $\theta_{68}$ value derived from a very preliminary angular resolution measurement, based on only a few $6.129 \mathrm{MeV}$ interactions, was $\sim 18 \mathrm{deg}$. This measurement is $\sim 2.5$ times greater that the kinematic limit. Agreement is quite good given that the electron track lengths were short and no correction was made for near-field parallax.

\subsection{3-DTI Prototype Development}

The development of the 3-DTI has been done in stages, our $10 \times 10 \times 15 \mathrm{~cm}^{3}$ and $30 \times 30 \times 15 \mathrm{~cm}^{3}$ versions are shown in Figure 7. A $30 \times 30 \times 7 \mathrm{~cm}^{3} 3$-DTI detector was used for an Office of Naval Research funded demonstration of neutron imaging [71] in an over-water environment. The 2-D readout for the 3-DTI detector used for neutron imaging did not require the two-stage GEM preamplifier because of the much higher specific ionization of protons compared to minimum ionizing electrons. We are in the process of expanding our mechanical support technique for the MWD+GEM to $10 \times 10 \mathrm{~cm}^{2}$ and $30 \times 30 \mathrm{~cm}^{2}$ MWDs. These larger prototypes will be used to 
make much more detailed and accurate angular resolution measurements than described above. These measurements and comparison with the calculations in this paper will be reported on in a subsequent publication.

\section{Design of the AdEPT Medium-Energy Pair Polarimeter}

The design of the AdEPT pair polarimeter has matured along with the development of the 3-DTI detector technology. Our baseline concept for the AdEPT instrument and spacecraft is described in Table 3.

Table 3 - AdEPT Instrument and Spacecraft Concept

\begin{tabular}{|ll|}
\hline Configuration: & 2 layers, $2 \times 2 \times 1 \mathrm{~m}^{3}$ 3-DTI modules \\
Ageom: & $2 \times 2 \mathrm{~m}^{2}=4 \times 10^{4} \mathrm{~cm}^{2}$ \\
Depth: & $200 \mathrm{~cm}$ \\
3-DTI Gas: & $\mathrm{Ar}(1100$ torr $)+\mathrm{CS}_{2}(40$ torr $)$ at $25 \mathrm{C}$ \\
3-DTI resolution: & $400 \mu \mathrm{m}$ in $\mathrm{x}, \mathrm{y}$, and $\mathrm{z}$ \\
Pressure vessel: & $\mathrm{Al}, \sim 5 \mathrm{~mm}$ thick, $\sim 300 \mathrm{~cm}$ diameter \\
Readout channels: & $\sim 40,000$ \\
Instrument power: & $\sim 500 \mathrm{~W}$ \\
Instrument mass: & $\sim 730 \mathrm{~kg}$ \\
Spacecraft: & zenith pointed, 3-axis stabilized \\
Orbit: & $28 \mathrm{deg}, \sim 550 \mathrm{~km}$ altitude \\
\hline
\end{tabular}

The total mass of the $\mathrm{Ar}+\mathrm{CS}_{2}$ gas is $\sim 20 \mathrm{~kg}$ at $25^{\circ} \mathrm{C}$ with a corresponding $\mathrm{RL}$ of $\sim 6.1 \times 10^{3} \mathrm{~cm}$. The 3-DTI drift field will be $\sim 1 \mathrm{kV} / \mathrm{cm}$ resulting in a negative drift velocity $v_{D}$ of $\sim 18 \mathrm{~mm} / \mathrm{ms}$. The 3-D spatial resolution is determined by the MWD pitch and the sampling frequency of the analogue signals from the MWD. The $400 \mu \mathrm{m}$ pitch of the MWD corresponds to a RMS resolution, $\sigma_{x, y}$ of $400 \mu \mathrm{m} / \sqrt{12} \approx 115 \mu \mathrm{m}$. Similar $z$ resolution of $400 \mu \mathrm{m}$ is determined by the digitization rate which, with a five-fold over sampling to avoid aliasing, is determined by the negative ion drift velocity and the digitization rate is $5 v_{D} / \sigma_{x, y} \approx 225 \mathrm{kHz}$.

The slow negative ion drift velocity reduces the value of a charged particle anti-coincidence detector or calorimeter in the AdEPT design. The time required for the ionization (track information) associated with a cosmic-ray or electron/positron pair to drift to the readout layer, the read-out delay, is tens of milli-seconds. Thus, a temporal coincidence between the track information and fast scintillator pulses is impractical. An exception to the long read-out delay is 
for those tracks which traverse the readout plane. In this case the ionization charge closest to the readout layer is read out in the next digitization period, $\sim 4 \mu$ s delay, and coincidence, in particular, with a calorimeter, may be practical.

In either case, an anti-coincidence signal cannot be used to discriminate cosmic rays from gamma-ray interactions since their ionization charge is in the gas and will be readout along with the gamma-ray track information. Thus, the readout delay results in a track "memory" in the TPC volume. In low-earth orbit, the integral SPENVIS ${ }^{3}$ isotropic cosmic ray proton flux is $\sim 56.6\left(\mathrm{~m}^{2} \mathrm{sr} \mathrm{s}\right)^{-1}$ above $\sim 6 \mathrm{GeV}$. The number of proton tracks crossing $1 \mathrm{~m}^{2}$ face of the TPC, with acceptance of $1 \pi \mathrm{m}^{2} \mathrm{sr}$, in a time corresponding to the maximum drift time, $1000 \mathrm{~mm} / \mathrm{V}_{\mathrm{d}}$, is $\sim 10$ tracks/face or $\sim 60$ tracks $/ \mathrm{m}^{3}$. The Gamma-ray interactions must be identified and discriminated from these tracks, using image recognition techniques. In separate work [72], a multi-core processor has been demonstrated and software is being developed to process the Giga-bit per second raw data from a $1 \mathrm{~m}^{3}$ TPC and separate the gamma-ray tracks from the CR tracks. Initial processing of simulated AdEPT data indicates that this separation, due to the high spatial information provided by the 3-DTI, is nearly lossless and result in little loss of effective area.

At this point in the development of the AdEPT concept we omit the anti-coincidence because it is not effective and do not include a calorimeter. A calorimeter could be added later, at the expense of increased mass, instrument complexity, and reduced instrument solid angle, if further instrument optimization and mission studies warrant.

\subsection{Effective area}

The performance of the AdEPT pair polarimeter has been calculated based on the concept parameters in Table 3, consideration of event reconstruction effects have not been included. The effective area of AdEPT is given by

$$
A_{\text {eff }}\left(E_{\gamma}\right)=A_{\text {geom }} \cdot\left[1-\exp \left(-\mu_{\text {pair }} \cdot \rho_{\text {gas }} \cdot \mathrm{D}\right)\right] .
$$

Where $A_{\text {geom }}$ is the TPC geometric area, $\mu_{\text {pair }}\left(E_{\gamma}\right)$ is the pair interaction coefficient as a

\footnotetext{
${ }^{3}$ Space Environment Information System https://www.spenvis.oma.be/
} 
function of gamma-ray energy in $\mathrm{cm}^{2} / \mathrm{g}, \rho_{\text {gas }}$ is the gas density in $\mathrm{g} / \mathrm{cm}^{3}$, and $\mathrm{D}$ is the depth of the TPC in $\mathrm{cm}$. The effective area of AdEPT is plotted in Figure 8 as a function of gamma-ray energy. The EGRET [73] and Fermi/LAT front [74] effective areas are shown for comparison.

\subsection{Continuum sensitivity}

The continuum sensitivity corresponding to a significance level, $n_{\sigma}$, is calculated from the expression for effective source counts [75]

$$
n_{\sigma}=S / \sqrt{S+B}
$$

Where $S$ and $B$ are, respectively, the number of source and background photons detected by an instrument with effective area $A_{\text {eff }}$, in observation time $T_{\text {obs }}$, and energy interval $\Delta E_{\gamma}$.

The easily recognized " $\wedge$ " signature of pair production results in detectors that are nearly free of instrumental background [47], thus the background counts are modeled using the all-sky average extragalactic gamma-ray emission spectrum derived from the EGRET data [76]

$$
F_{B}\left(E_{\gamma}\right)=7.32 \times 10^{-9}\left(E_{\gamma} / 451 \mathrm{MeV}\right)^{-2.10} \text { photons } \mathrm{cm}^{-2} \mathrm{~s}^{-1} \mathrm{sr}^{-1} \mathrm{MeV}^{-1} .
$$

We choose to use the flatter EGRET diffuse spectrum since it is consistent with the COMPTEL [77] and SAS-2 [78] results rather than the steeper Fermi spectrum [79], which if extrapolated down to the medium-energy region is inconsistent with the COMPTEL results.

The number of background counts is given by

$$
B\left(E_{\gamma}\right)=F_{B}\left(E_{\gamma}\right) \cdot A_{\mathrm{eff}}\left(E_{\gamma}\right) \cdot T_{\mathrm{obs}} \cdot \Omega\left(E_{\gamma}\right) \cdot \Delta E_{\gamma} \text { photons, }
$$

where

$$
\Omega\left(E_{\gamma}\right)=2 \pi\left(1-\cos \theta_{68}\left(E_{\gamma}\right)\right) \mathrm{sr}
$$

is the solid angle containing $68 \%$ of the photons from a point source. The number of source counts corresponding to detection significance is determined by solving Eq. (6) for $S\left(E_{\gamma}\right)$ and taking the positive root.

$$
\mathrm{S}\left(\mathrm{E}_{\gamma}\right)=\frac{1}{2}\left(\mathrm{n}_{\sigma}^{2}+\sqrt{\mathrm{n}_{\sigma}^{4}+4 \mathrm{~B}\left(\mathrm{E}_{\gamma}\right) \mathrm{n}_{\sigma}^{2}}\right) \approx \mathrm{n}_{\sigma}^{2}+\mathrm{B}\left(\mathrm{E}_{\gamma}\right) .
$$

The corresponding differential continuum source flux or sensitivity is given by 


$$
F_{\mathrm{S}}\left(E_{\gamma}\right)=S\left(E_{\gamma}\right) /\left(A_{\mathrm{eff}} \cdot T_{\mathrm{obs}} \cdot \Delta E_{\gamma} \cdot 0.68\right) \text { photons } \mathrm{cm}^{-2} \mathrm{~s}^{-1} \mathrm{MeV}^{-1}
$$

where the factor of 0.68 in the denominator corresponds to the use of $\theta_{68}$ in Eq. 8. The AdEPT $3 \sigma$ continuum source sensitivity multiplied by $E_{\gamma}^{2}$ calculated on the assumptions of an observation time of $10^{6} \mathrm{~s}$ and $\Delta E_{\gamma}=E_{\gamma}$ is shown in Figure 9. The sensitivity for EGRET and Fermi-LAT front, calculated on the same assumptions are shown for comparison. The AdEPT sensitivity is better than Fermi up to $\sim 200 \mathrm{MeV}$, as desired.

The AdEPT sensitivity calculated here must be considered as a lower limit since the effective area calculation does not include any corrections for interactions near the edge of the TPC or inefficiencies in the track recognition. Further, the sensitivity for sources near the Galactic center will be up to an order of magnitude higher, since the 30 to $100 \mathrm{MeV}$ Galactic diffuse emission is about an order of magnitude higher than the extragalactic emission [80]. The assumption of low instrumental background may also be optimistic, since, without an anticoincidence, neutral pions generated by cosmic-ray protons interacting with the pressure vessel are a potential source of background. This will be taken into account as part of the detailed instrument simulations.

\subsection{Minimum detectable polarization}

The minimum detectable polarization (MDP) for a given instrument can be written as [81] :

$$
\operatorname{MDP}\left(E_{\gamma}\right)=n_{\sigma} / \lambda \sqrt{S+B} / S
$$

where $\mathrm{S}$ and B are the observed source and background counts and the asymmetry factor, is defined in Eq. 4. The asymmetry factor for co-planner events has been calculated over the entire energy range allowing for screening of the nucleus by Kel'ner et al. [26] and above $10 \mathrm{MeV}$ using Monte Carlo integration by Depaola and Kozemeh [82]. The Kel'ner value rises rapidly from zero at $1 \mathrm{MeV}$ to a maximum of $\sim 0.46$ at $\sim 2 \mathrm{MeV}$ and then decreases to a high energy asymptotic value of $\sim 0.4$. The Depaola and Kozemeh value is $\sim 0.12$ at $10 \mathrm{MeV}$ and rises to an asymptotic value of $\sim 0.2$. The difference in asymptotic values may be due to different assumptions made in the calculations. Depaola and Kozemeh also find that the asymmetry factor also changes sign for events with small deviations from co-planarity, thus to obtain a more accurate value of the asymmetry factor it will be necessary to include the instrument angular 
resolution in the integration. For this work we adopt a conservative fixed value for the asymmetry factor $\lambda=0.15$ for $\mathrm{E}_{\gamma}>5 \mathrm{MeV}$ to evaluate the MDP for a source with spectrum and intensity equal to the Crab nebula [83] (defined as a "1 Crab" source). Assuming equal energy split between the electron and positron, the MDP for $1 \mathrm{Crab}, 100 \mathrm{mCrab}$ and $10 \mathrm{mCrab}$ sources is shown in Figure 10.

\subsection{Energy resolution}

The high spatial resolution of the 3-DTI tracker allows multiple Coulomb scattering to be used to estimate the energy $(p v)$ of electrons above $\sim 1 \mathrm{MeV}$ which will generally exit the 3-DTI gas volume. Specific ionization, $d E / d x$, and residual range can be used for lower energy and stopping electrons. The techniques developed to determine the energy of particles leaving tracks in photographic emulsions [84][85][86] have also been used with bubble chambers [87]. These techniques have been extended to include Kalman filtering [88] and been used to measure the through-going particle momentum in the ICARUS T600 TPC [89]. An estimate of the AdEPT energy resolution can be obtained by scaling the ICARUS muon momentum by the square root of the ratio of the detector RLs, i.e. $\sqrt{X_{0, \mathrm{~L}-\mathrm{Ar}} / X_{0, \mathrm{G}-\mathrm{Ar}}} \approx 29.4$. This approach is valid because the spatial resolution per $\mathrm{RL}$ of AdEPT, $\left(1.2 \times 10^{4} \mathrm{~cm} / \mathrm{RL}\right) /(0.04 \mathrm{~cm} / \mathrm{sample})=$ $3.0 \times 10^{5}$ samples/RL, is much higher than for ICARUS, $(14 \mathrm{~cm} / \mathrm{RL}) /(0.3 \mathrm{~cm} / \mathrm{sample})=$ 46.7 samples/RL. The scaled ICARUS simulations using the classical and Kalman filter methods, shown in Figure 11 with the scaled electron momentum, indicates that the expected AdEPT momentum resolution will be better than $\sim 15 \%$ for electron momenta above $\sim 10 \mathrm{MeV}$. The much higher resolution of AdEPT may result in improved low momentum resolution compared to ICARUS. Monte-Carlo simulations similar to those done for ICARUS are being done for the AdEPT instrument and the results will be presented in a future paper.

\section{Summary}

The AdEPT instrument concept based on the 3-DTI gas TPC technology (Table 3) will provide unique observations in the 5 to $200 \mathrm{MeV}$ energy range. These observations with angular resolution within a factor of two of the pair production kinematic limit and minimum detectable polarization $<2 \%$ for a $100 \mathrm{mCrab}$ source up to $\sim 150 \mathrm{MeV}$ will address a wide range of the critical science goals. The calculated AdEPT performance is encouraging and the few instrument challenges are readily tractable. Detailed Geant 4 simulations will be completed to confirm these 
calculations.

Milestones in the AdEPT development program include testing of $10 \times 10 \times 15 \mathrm{~cm}^{3}$ and $30 \times 30 \times 30 \mathrm{~cm}^{3} 3$-DTI detectors. Currently proposed work will build a $50 \times 50 \times 100 \mathrm{~cm}^{3} 3$-DTI prototype of the AdEPT instrument module. Future work will include calibration of this AdEPT module prototype at the Duke University HIGS accelerator that offers $100 \%$ polarized gammarays from $\sim 15$ to $50 \mathrm{MeV}$ [90], and a balloon flight in the 2018-20 time-frame. The goals of the accelerator calibration will be to determine the optimum electron energy determination algorithms, gamma-ray direction and energy, and the energy dependent polarization modulation factor, and to verify the angular and energy distributions for pair production near threshold simulated with Geant4. The balloon flight will confirm that gamma-rays can be identified in the presence of a high charge particle background.

We envision AdEPT, a future space mission, to be the next step in observational gamma-ray astrophysics that will open up a new window in medium-energy gamma-ray astrophysics with its unique capability to measure polarization. . 


\section{ACRONYMES}

$\begin{array}{ll}\text { 3-DTI } & \text { Three Dimensional Track Imager } \\ \text { AdEPT } & \text { Advanced Energetic Pair Telescope } \\ \text { GEM } & \text { Gas Electron Multiplier } \\ \text { MCS } & \text { Multiple Coulomb Scattering } \\ \text { MDP } & \text { Minimum Detectable Polarization } \\ \text { mRL } & \text { milliradiation Length } \\ \text { MWD } & \text { Micro-Well Detector } \\ \text { NI-TPC } & \text { Negative Ion TPC } \\ \text { SSD } & \text { Silicon Strip Detector } \\ \text { TPC } & \text { Time Projection Chamber }\end{array}$

REFERENCES

[1] M. Tavani, et al. 2003, The AGILE instrument, Proc. SPIE 4851, X-Ray and Gamma-Ray Telescopes and Instruments for Astronomy, 1151 (March 10, 2003); doi: 10.1117/12.461312

[2] P.F. Michelson, 2003, Instrumentation for the Gamma-ray Large Area Space Telescope (GLAST) mission, Proc. SPIE 4851, X-Ray and Gamma-Ray Telescopes and Instruments for Astronomy, 1144 (March 10, 2003); doi:10.1117/12.461541

[3] W. Cui, 2009, TeV gamma-ray astronomy, Research in Astron. Astrophys. 9, No. 8, 841-860

[4] C.E. Fichtel, et al. 1976, Significance of Medium-Energy Gamma-Ray Astronomy to the Study of Cosmic Rays, ApJ, 208, 211-219

[5] F.W. Stecker, 2013, Constraining Superluminal Electron and Neutrino Velocities using the 2010 Crab, Nebula Flare and the IceCube PeV Neutrino Events, arXiv:1306.6095.

[6] R.D. Blandford, 1976, Accretion disc electrodynamics - A model for double radio sources, MNRAS 176, 465

[7] R.V.E. Lovelace, 1976, Dynamo model of double radio sources, Nature 262, 649

[8] R.A. Laing, 1981, Magnetic fields in extragalactic radio sources, Ap. J. 248, 87-104 
[9] H. Kigure, et al. 2004, Distribution of Faraday Rotation Measure in Jets from Active Galactic Nuclei. II. Prediction from Our Sweeping Magnetic Twist Model for the Wiggled Parts of Active Galactic Nucleus Jets and Tails, Ap. J. 608, 119-135

[10] W. Coburn, \& S.E. Boggs, 2003, Polarization of the prompt gamma-ray emission from the gamma-ray burst of 6 December 2002, Nature, 423, 415

[11]D. Gotz, 2013, INTEGRAL Results on Gamma-Ray Bursts, in An INTEGRAL view of the high-energy sky (the first 10 years), Proceedings of Science (editors: F. Lebrun, A. Goldwurm and C. Winkler), arXiv:1302.4847v1

[12]D. Yonetoku, et al. 2011, Detection of Gamma-Ray Polarization in Prompt Emission of GRB 100826A, Ap. J. Let. arXiv:1111.1779v1

[13]F.W. Stecker, Tests of Lorentz Invariance Using High-Energy Astrophysics Observations, 2011 Astropart. Phys. 35,95

[14] V.A. Kostelecky, \& M. Mewes, 2013, Constraints on relativity violations from gamma-ray bursts, Phys. Rev. Lett. 110 201601, arXiv:1301.5367

[15]M. Forot, et al., 2008, Polarization of the Crab Pulsar and Nebula as Observed by the INTEGRAL/IBIS Telescope, Ap. J. L29, 688

[16]F.W. Stecker \& M.H. Salamon, 2001, The Extragalactic Gamma-ray Background, AIP Conf. Proc. 587, 432, astrop-ph/0104368

[17] J.I. Trombka, et al. 1977, Reanalysis of the Apollo cosmic gamma-ray spectrum in the 0.3 to $10 \mathrm{MeV}$ energy region, Ap. J. 212, 925-935

[18] S.C. Kappadath, et al. 1995, The Preliminary Cosmic Diffuse Gamma-Ray Spectrum from $800 \mathrm{keV}$ to $30 \mathrm{MeV}$ Measured with COMPTEL, 24th ICRC (Rome) 2, 230-233

[19]R. Jost, J.M. Luttinger, \& M. Slotnick, 1950, Distribution of Recoil Nucleus in Pair Production by Photons, Phys. Rev. 80, 189-96

[20] D. Bernard, 2013, TPC in భray astronomy above pair-creation threshold, Nucl. Instr. Meth. A 701, 225-230

[21] G.O. Depaola \& M.L Iparraguirre, 2009, Angular distribution for the electron recoil in pair production by linearly polarized gamma-rays on electrons, Nucl. Inst. Meth. A 611 84-92

[22] S.M. Derdeyn, et al. 1972, The Small Astronomy Satellite-2 (SAS-2) gamma-ray telescope, Nucl. Instr. Meth. 98,557

[23] Bignami, G. F., et al. 1975, The COS-B experiment for gamma-ray astronomy, Space Sci. Instrum. 1, 245

[24] Kanbach, G., et al. 1988, The project EGRET (Energetic Gamma-Ray Experiment Telescope) on NASA's Gamma-Ray Observatory GRO, Space Sci. Rev. 49, 69-84 
[25] V.I. Kryshkin, A.G. Sterligov, \& Yu.P. Usov, 1969, Production of electron-positron pairs in a nuclear coulomb field, Russian Physics Journal, 13, no. 6, 771-772

[26] S.R. Kel'ner, Yu.D. Kotov, \& V.M. Logunov, 1975, Methods of measuring linear polarization of gamma quanta, Sov. J. Nucl. Phys. 21, 313-315

[27]D.A. Kniffen, et al. 1978, Observations of Medium-Energy Gamma-Ray Emission from the Galactic Center Region, Ap. J. 225, 591-8

[28]R.W. Ross, et al. 1969, A Gamma-Ray Telescope Utilizing Large Area Wire Spark Chambers, IEEE Transactions, NS-16, 304

[29] G. Kanbach, et al. 2005, Development and Calibration of the tracking Compton/Pair Telescope MEGA, Nucl. Instr. Meth. A 541, 310-322

[30] T.J. O'Neill, et al. 2001, The TIGRE gamma-ray telescope, AIP Conf. Proc. 587, 882

[31] A.D. Zych, et al. 2000, 10-100 MeV Gamma-Ray Astronomy with GLAST, AAS HEAD, 32, 1263; NASA Final Report, NRA 98-217-02 (1999).

[32] W.R. Innes, 1993, Some formulas for estimating tracking errors, Nucl. Instr. Meth. A 239 238-242

[33] G. Kanbach, et al, 1988, The Project EGRET on NASA's GRO, Space Sci. Rev. 49 69-84

[34] J.E. McEnery, et al. 2012, Fermi Gamma-Ray Space Telescope, Optical Engineering, 51 (1) 011012

[35] A. Bulgarellia, 2010, The AGILE silicon tracker: Pre-launch and in-flight configuration, Nucl. Instr. Meth. A $614,213-226$

[36] V.L. Highland, 1975, Some practical remarks on multiple scattering, Nucl. Instr. Meth. 129, 497, and 1979, Erratum, Nucl. Instr. Meth. 161, 171

[37] G.R. Lynch \& O.I Dahl, 1991, Approximations to multiple Coulomb scattering, Nucl. Instr. Meth. B 58, 6-10

[38] D. Bernard, 2013, Corrigendum to TPC in $\gamma$-ray astronomy above pair-creation threshold, Nucl. Instr. Meth. A $713,76-77$

[39]H. Sun, Z. Xu, \& C. Gao, 2013, Multifunctional, Ultra-Flyweight, Synergistically Assembled Carbon Aerogels, Advanced Materials, 25, no. 18, 2554-2560, DOI: 10.1002/adma.201204576

[40]C.N. Yang, 1950, Possible Experimental Determination of Whether the Neutral Meson is Scalar or Pseudoscalar, Phys. Rev. 77, 722-723

[41] G.C. Wick, 1951, Determination of gamma-ray polarization by pair production, Phys. Re. 81, 467-468

[42] L.C. Maximon, \& H. Olsen, 1962, Measurement of Linear Photon Polarization by Pair Production, Phys. Rev. $126,310-319$ 
[43] J.R. Mattox, H.A. Mayer-Hassewander, \& A.W. Strong, 1990, Analysis of the COS B Data for Evidence of Linear Polarization of Vela Pulsar Gamma Rays, Ap. J. 363, 270-273

[44] J.R. Mattox, 1991, The sensitivity of EGRET to gamma-ray polarization, Exper. Astro. 2, 75-84

[45]R. Buehler, et al., Measuring polarization of gamma-rays with Fermi, SciNeGHE 2010 Trieste, http://scineghe2010.ts.infn.it/programmaScientifico.php

[46]P. Rice-Evans, 1974, Spark, streamer, proportional and drift chambers, Richelieu Press, London

[47] R.C. Hartman, 1989, Astronomical Gamma Ray Telescopes in the Pair Production Regime, Nucl. Phys. B Proc. Suppl. 10, 130-138

[48] P.F. Bloser, et al. 2005, Gas Micro-well Track Imaging Detector for Gamma-ray Astronomy, in UV, X-Ray, and Gamma-Ray Space Instrumentation for Astronomy XIV (San Diego, CA: SPIE), 5898, 152-163

[49] P.F. Bloser, et al. 2004, A Concept for a High-Energy Gamma-ray Polarimeter, SPIE 5165 322-333

[50] S.D. Hunter, et al. 2010, Development of the Advanced Energetic Pair Telescope (AdEPT) for Medium-Energy Gamma-Ray Astronomy, SPIE Space Telescopes and Instrumentation, 7732:773221

[51] S.D. Hunter, et al. 2012, Development of a telescope for medium energy gamma-ray astronomy, Proceedings of SPIE Vol. 8443, 84430F

[52] K. Ueno, et al. 2011, Development of the tracking Compton/pair-creation camera based on a gaseous TPC and a scintillator camera, Nucl. Instr. Meth. A 628, 158

[53]D.R. Nygren \& J.N. Marx, 1978, The Time Projection Chamber, Physics Today, 31, 46

[54] D. Attie, 2009, TPC Review, Nucl. Instr. Meth. A 598, 89

[55] C.J. Martoff, et al. 2000, Suppressing drift chamber diffusion without magnetic field, Nucl. Instr. Meth. A 440, 355-359

[56] R. Bellazzini, et al. 1999, The WELL detector, Nucl. Instr. Meth. A 423, 125-134

[57] G. Chaplier, et al. 1999, Preliminary results of the experimental and simulated intrinsic properties of the Compteur A Trou (CAT) detector: behavior with synchrotron radiation, Nucl. Instr. Meth. A 426, 339-355

[58] W.K. Pitts, et al. 1999, Development and operation of laser machined microwell detectors, Nucl. Instr. Meth. A $438,277-281$

[59] P. Deines-Jones, et al. 2002, Imaging micro-well proportional counters fabricated with masked UV laser ablation, Proc. of the $5^{\text {th }}$ Position Sensitive Detector Workshop, Nucl. Instr. Meth. A 477, 55-58

[60]P. Deines-Jones, et al. 2002, Large-area imaging micro-well detectors for high-energy astrophysics, Nucl. Instr. Meth. A 478, 130-134 
[61]F. Sauli, 1997, GEM: a new concept for electron multiplication in gas detectors, Nucl. Instr. Meth. A 386, 531534; A GEM of a detector, CERN Courier, cerncourier.com/cws/article/cern/27921

[62] L.B. Loeb, 1955, Basic processes of gaseous electronics, Univ. of California, Berkeley, CA

[63]F. Piuz, 1983, Measurements of the longitudinal diffusion of a single electron in gas mixtures used in proportional counters, Nucl. Instr. Meth. A 205, 425-36

[64] H.R. Crane, 1961, $\mathrm{CO}_{2}$ - $\mathrm{CS}_{2}$ Geiger counter, Rev. Sci. Inst. 32, 953

[65] M.P. Dion, et al. 2011, Negative ion drift velocity and longitudinal diffusion in mixtures of carbon disulfide and methane, Nucl. Instr. Meth. A 648, 186-91

[66] M. Zubek, J. Gackowska, \& A. Snegursky, 2003, Dissociative excitation of carbon disulfide by electron impact, Radiation Physics and Chemistry 68 323-328

[67] T. Ohnuki, et al. 2001, Measurement of carbon disulfide anion diffusion in a TPC, Nucl. Instr. Meth. A 463, $142-151$

[68] M.P. Dion, et al. 2010, On the mechanism of Townsend avalanche for negative molecular ions, Astro. Phy., 33, 216-220

[69] Yu. Ivaniouchenkov, et al. 1999, Breakdown limit studies in high-rate gaseous detectors, Nucl. Instr. Meth. A 422 300-304

[70] A. Bressan, et al. 1999, High rate behavior and discharge limits in micro-pattern detectors, Nucl. Instr. Meth. A $424,321-342$

[71] S. Son, et al. 2010, Neutron Imaging Camera, IEEE/NSS, Knoxville, TN, N11-3

[72] T. Sheets, et al. 2014, A multi-core processor for the Advanced Energetic Pair Telescope, in preparation.

[73]D.J. Thompson, et al. 1993, Calibration of the Energetic Gamma-Ray Experiment Telescope (EGRET) for the Compton Gamma-Ray Observatory, Ap. J. Sup. 86, 629-656

[74] M. Ackermann, et al. 2012, The Fermi Large Area Telescope On Orbit: Event Classification, Instrument Response Functions, and Calibration, Ap. J.S 203 4, doi:10.1088/0067-0049/203/1/4, arXiv:1206.1896v2

[75] G. Quittner, 1964, Intensity, background and resolution width in nuclear counting experiments, Nucl. Inst. \& Meth. 31, 61-67

[76] P. Sreekumar, et al. 1998, EGRET observations of the extragalactic diffuse gamma ray emission, Ap. J. 494, 523-534

[77] S.C. Kappadath, et al. 1996, The preliminary cosmic diffuse $\gamma$-ray spectrum from $800 \mathrm{keV}$ to $30 \mathrm{MeV}$ measured with COMPTEL, A\&AS, 120, 619-622 
[78]D.J. Thompson \& C.E. Fichtel, 1982, Extragalactic gamma radiation - Use of galaxy counts as a galactic tracer, A\&A, 109, 352-354

[79] A. Abdo, et al. 2010, Spectrum of the Isotropic Diffuse Gamma-Ray Emission Derived from First-Year Fermi Large Area Telescope Data, Phys.Rev.Lett.104:101101

[80] S.D. Hunter, et al. 1997, EGRET Observations of the Diffuse Gamma-Ray Emission from the Galactic Plane, Ap. J. 481, 205-240

[81]R. Novick, 1975, Stellar and solar x-ray polarimetry, Space Sci. Rev. 18, 389-408

[82] G.O. Depaola \& C.N. Kozameh, 1998, Detecting polarized gamma-rays by pair production, Rad. Phys. Chem. $53,455-459$

[83]L. Kuiper et al. 2001, The Crab pulsar in the 0.75-30 MeV range, A coherent high-energy picture from soft Xrays up to high-energy gamma-rays, A\&A 378 918-935

[84] H.S. Snyder and W.T. Scott, 1949, Multiple scattering of fast charged particles, Phys. Rev. 76, 220-225; W.T. Scott and H.S. Snyder, 1950, On scattering induced curvature for fast charged particles, Phys. Rev. 78, 223230

[85] P.H. Fowler, 1950, Nuclear transmutations produced by cosmic-ray particles of great energy, Phil. Mag. Ser. 7, $41,169-184$

[86]D.S. Schonland, 1952, On the utilization of multiple scattering measurements, Proc. Phys. Soc. A 65, 640-656

[87]R.L. Gluckstern, 1963, Uncertainties in track momentum and direction, due to multiple scattering and measurement errors, Nucl. Instr. Meth. 24, 361-389

[88]R. Frühwirth, 1987, Application of Kalman filtering to track and vertex fitting, Nucl. Instr. Meth. A 262, 444450

[89] A. Ankowski, et al., 2006, Measurement of through-going particle momentum by means of multiple scattering with the ICARUS T600 TPC, Eur. Phys. J. C48, 667-676; 10.1140/epjc/s10052-006-0051-3; arXiv:hepex/0606006

[90] Duke University HIGS accelerator, www.tunl.duke.edu/web.tunl.2011a.higs.php 


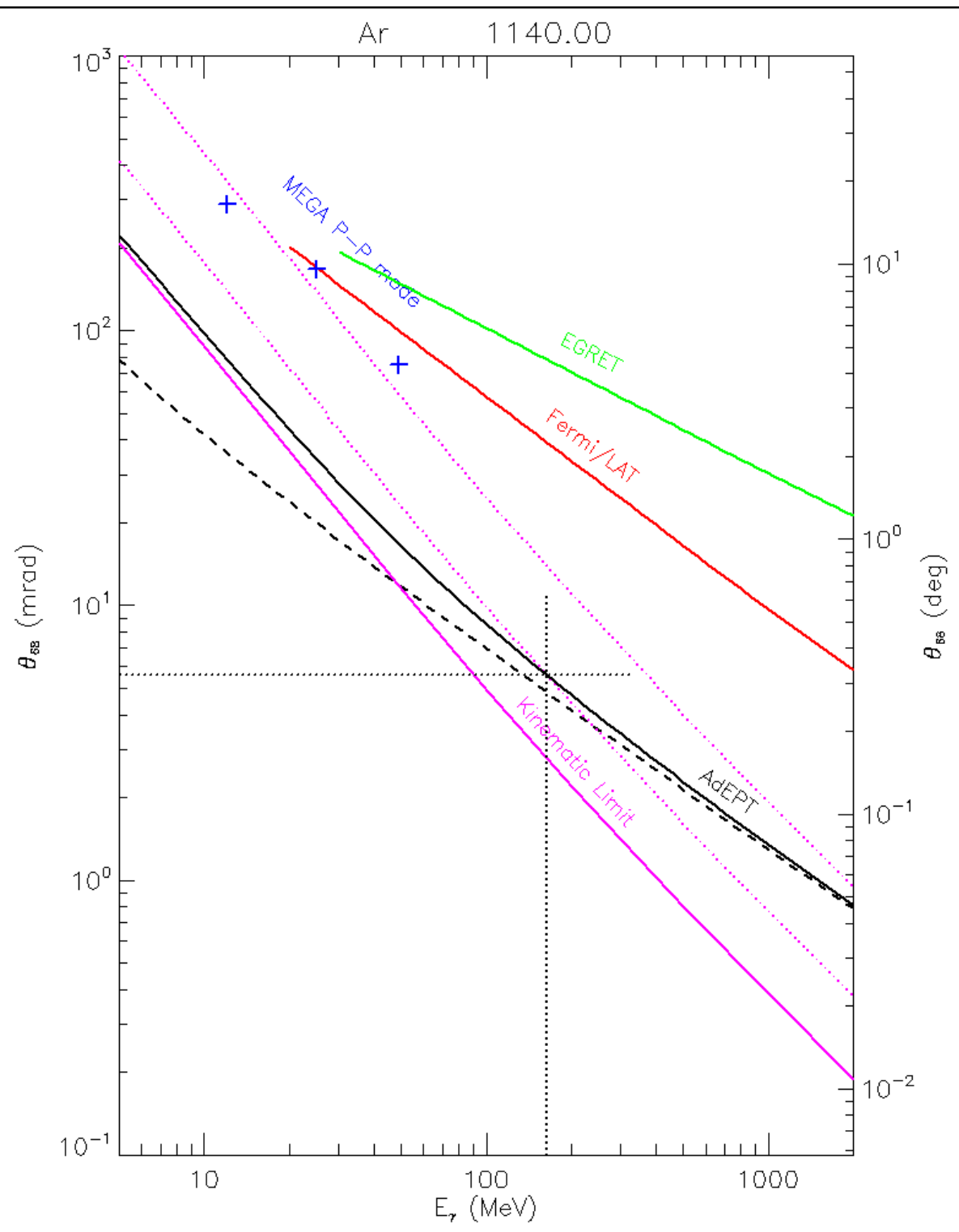

Figure 1 - The calculated angular resolution of the AdEPT telescope concept (solid black line, see $\$ 6$ and Table 3) as a function of the gamma ray energy is the quadrature sum of the kinematic limit determined for nuclear pair production from [19] (solid magenta line) and the angular resolution limited only by MCS of the electron-positron pair (black dashed line). Twice, and five times the kinematic limit is also shown (dotted magenta lines). Below $\sim 200 \mathrm{MeV}$, the AdEPT telescope will achieve angular resolution within a factor two of the kinematic limit. The MEGA [29] measured pair production angular resolution (blue crosses), EGRET [73] calibrated angular resolution (green line), and Fermi/LAT front [74] on-orbit angular resolution (red line) are shown for comparison. 


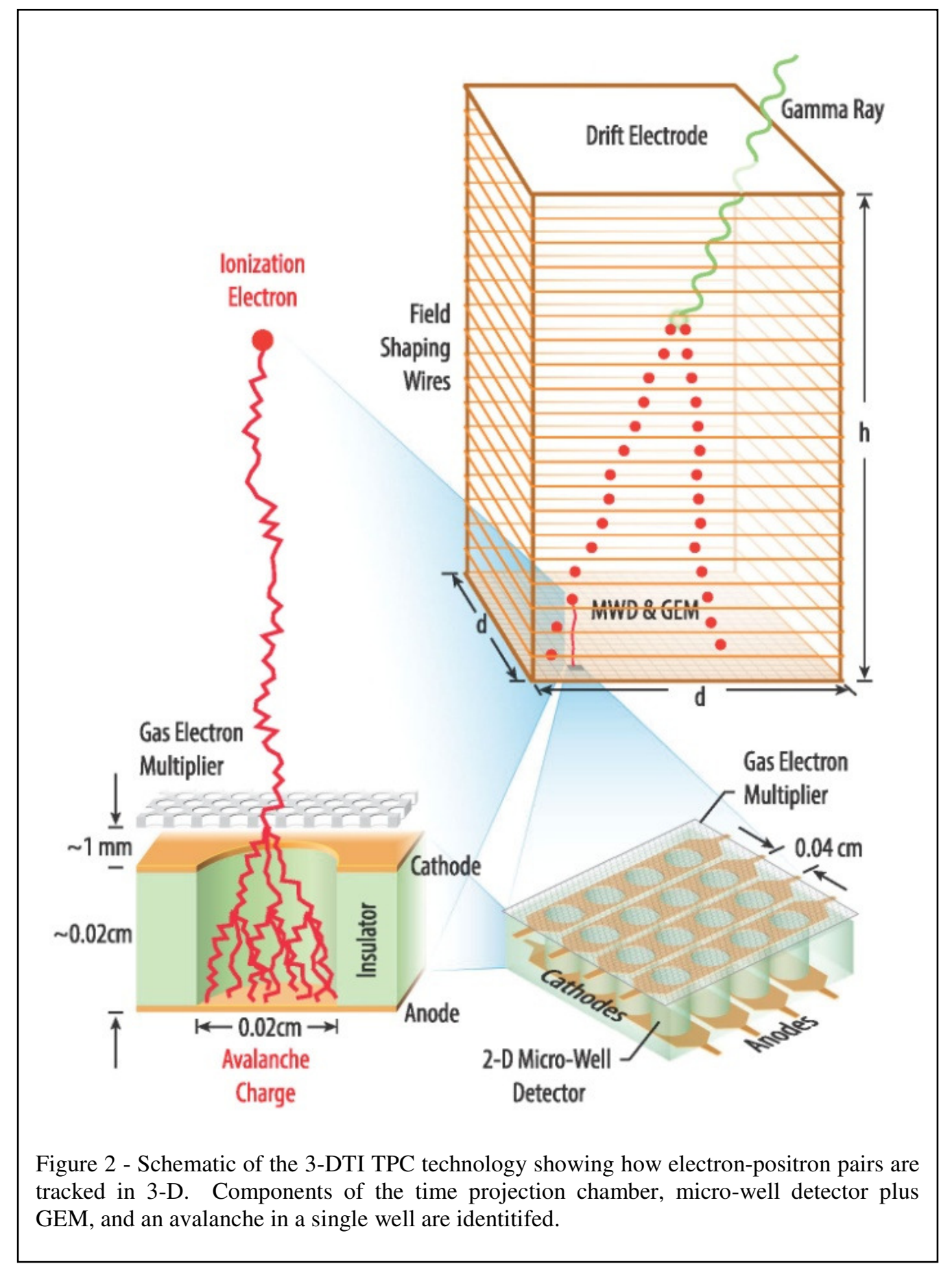




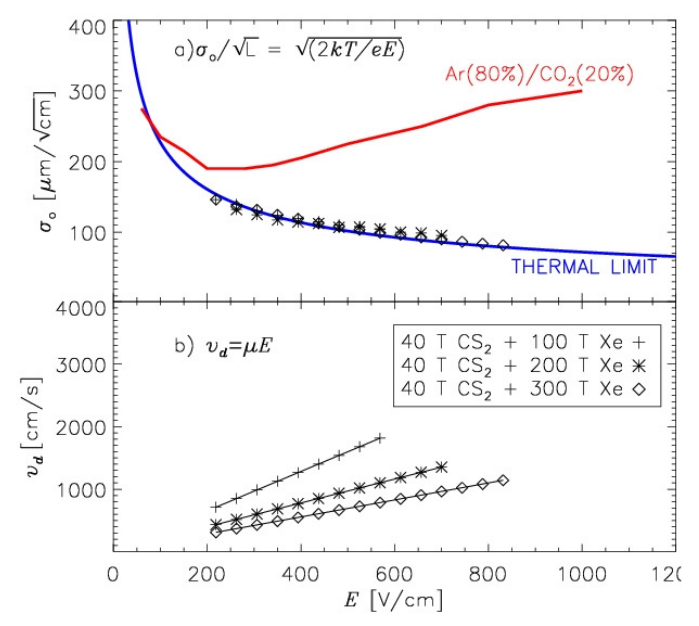

Figure 3 - (top) The longitudinal negative ion diffusion and (bottom) the drift velocity $\left(v_{d}=\mu \mathrm{E}\right)$ for mixture of $\mathrm{Xe}+\mathrm{CS}_{2}$. Thermal diffusion limit for $\mathrm{T}=300 \mathrm{~K}$ is shown as the blue line. Non-thermal diffusion for $\mathrm{Ar}+\mathrm{CO}_{2}[63]$ is shown as the red line.

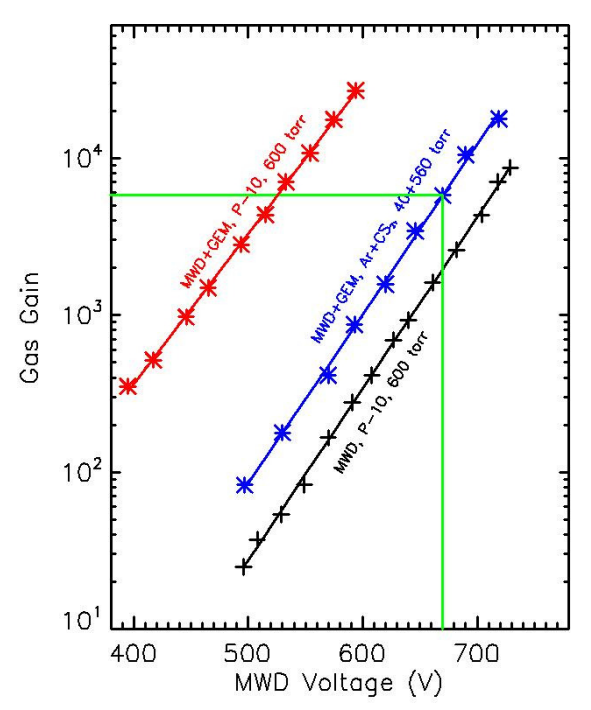

Figure 4 - The gain of our MWD (black data and line) and MWD+GEM (red data and line) as a function of MWD voltage measured in P-10 at 600 torr. The GEM pre-amplifier was operated at a gain of $\sim 100$ corresponding to a MWD voltage reduction of $\sim 200 \mathrm{~V}$. The gain of the MWD+GEM as a function of MWD voltage measured in $\mathrm{Ar}+\mathrm{CS}_{2}$ (blue data and line) shows that gains in excess of $10^{4}$ can be achieved. The nominal operating voltage for the MWD and the total gain are indicated by the green lines. 


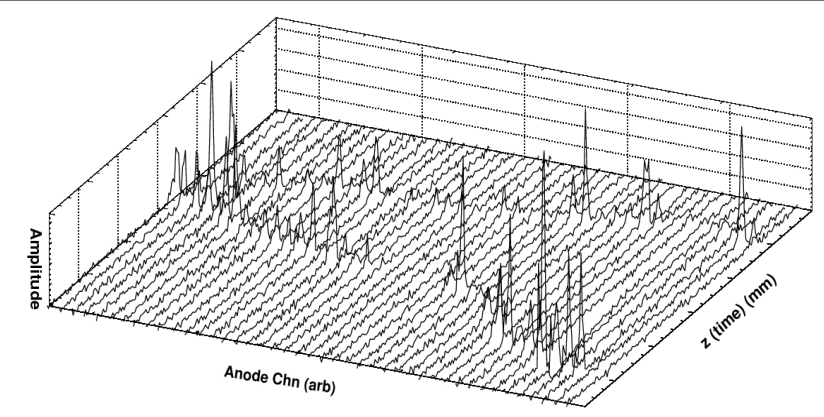

Figure 5 - Electron-positron tracks (X-Z projection) from a 6.129 MeV gamma ray pair interaction in 3-DTI.

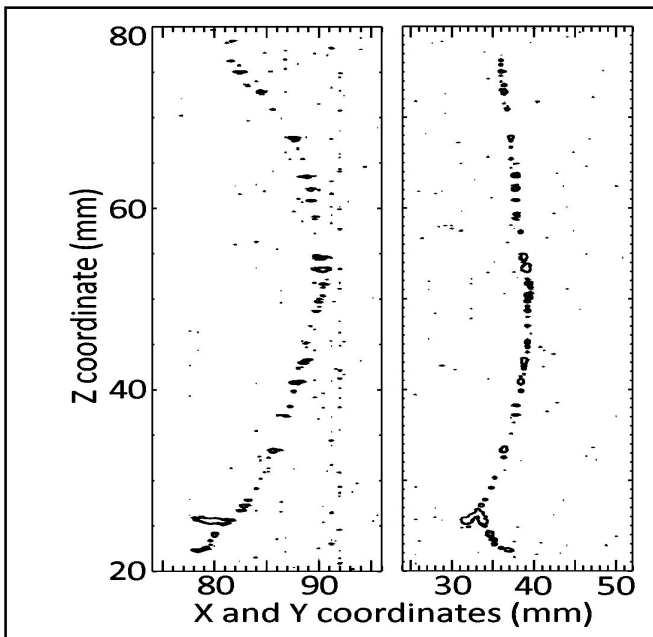

Figure $6-3$-DTI electron track from ${ }^{90} \mathrm{Sr}$ in $\mathrm{Ar}+\mathrm{CS}_{2}(560+40$ torr $)$ using a MWD+GEM operating at a total gain of $10^{4}$. The left and right images are the $\mathrm{X}-\mathrm{Z}$ and $\mathrm{Y}-\mathrm{Z}$ projections of the electron track. 

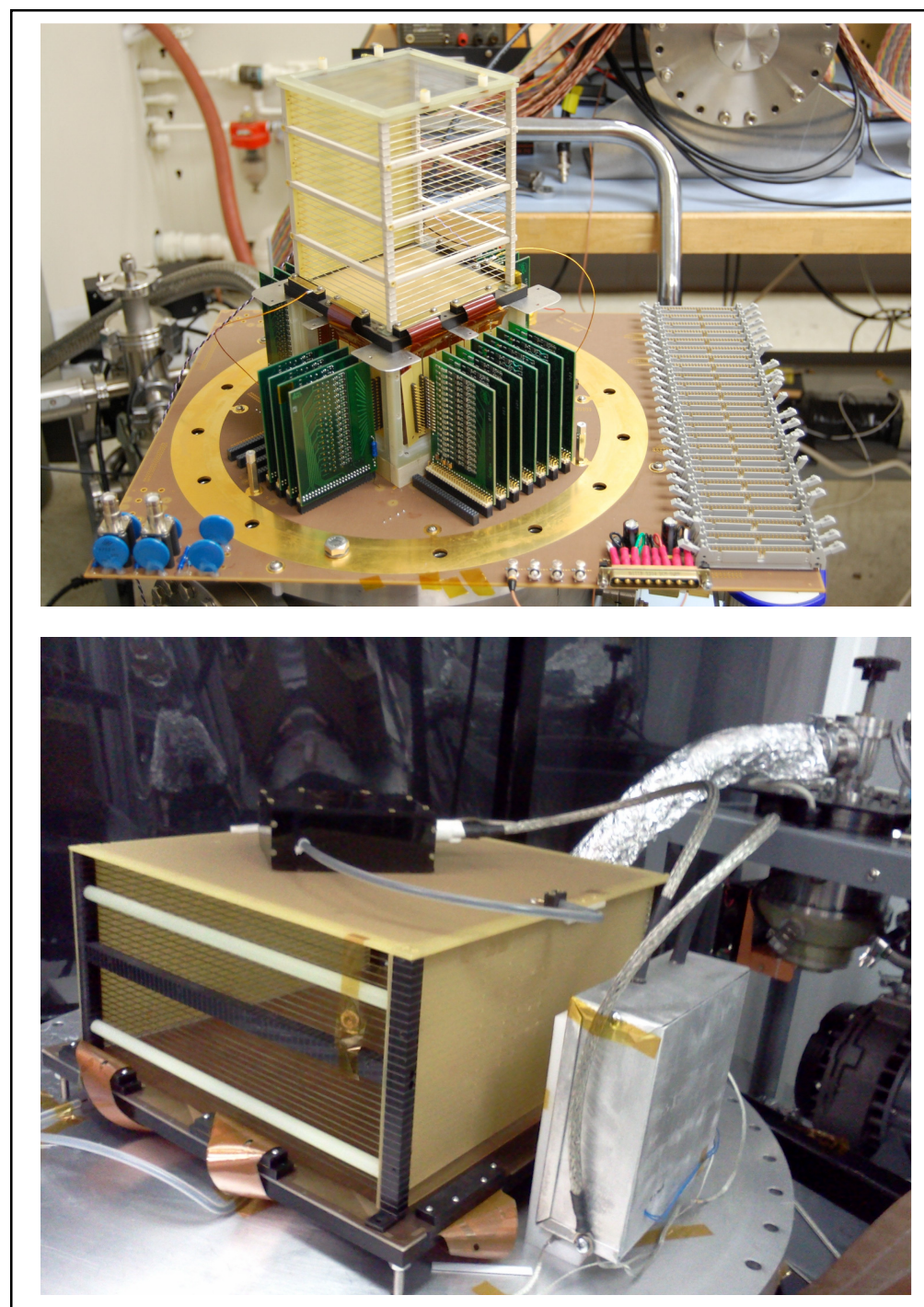

Figure 7 - (top) $10 \times 10 \times 15 \mathrm{~cm}^{3} 3$-DTI detector consisting of a $10 \times 10 \mathrm{~cm}^{2}$ WMD and $15 \mathrm{~cm}$ tall TPC volume. Eleven of the 32 total 16-channel discrete front-end electronics cards are visible. (bottom) $30 \times 30 \times 15 \mathrm{~cm}^{3}$ detector. Three of the 8 flex circuits that connect the MWD to the front-end electronics are visible as well as the drift field high-voltage power (black box) and ripple filter (aluminum box). The upper portions of the vacuum chambers for both detectors were removed for these photos. 


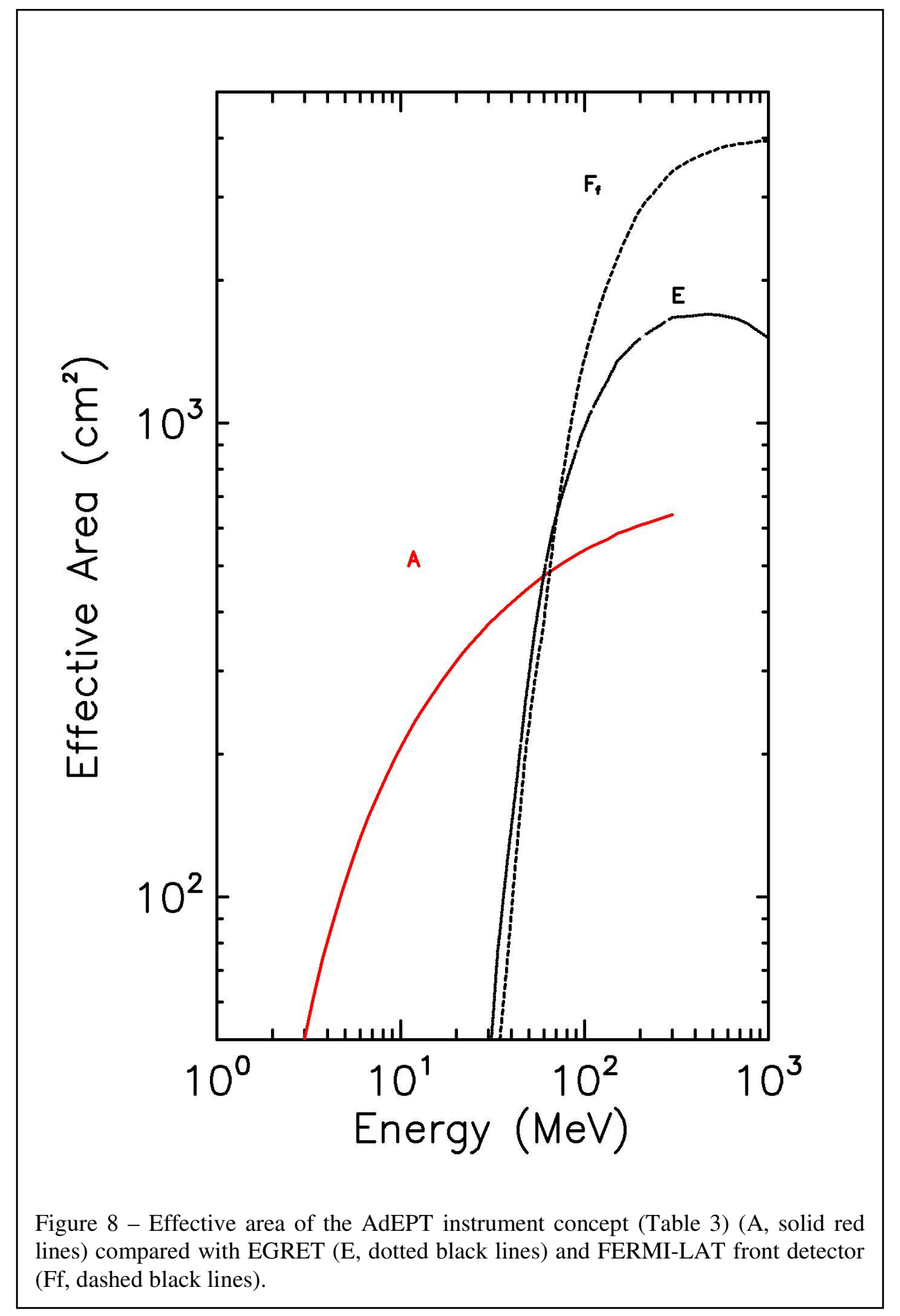




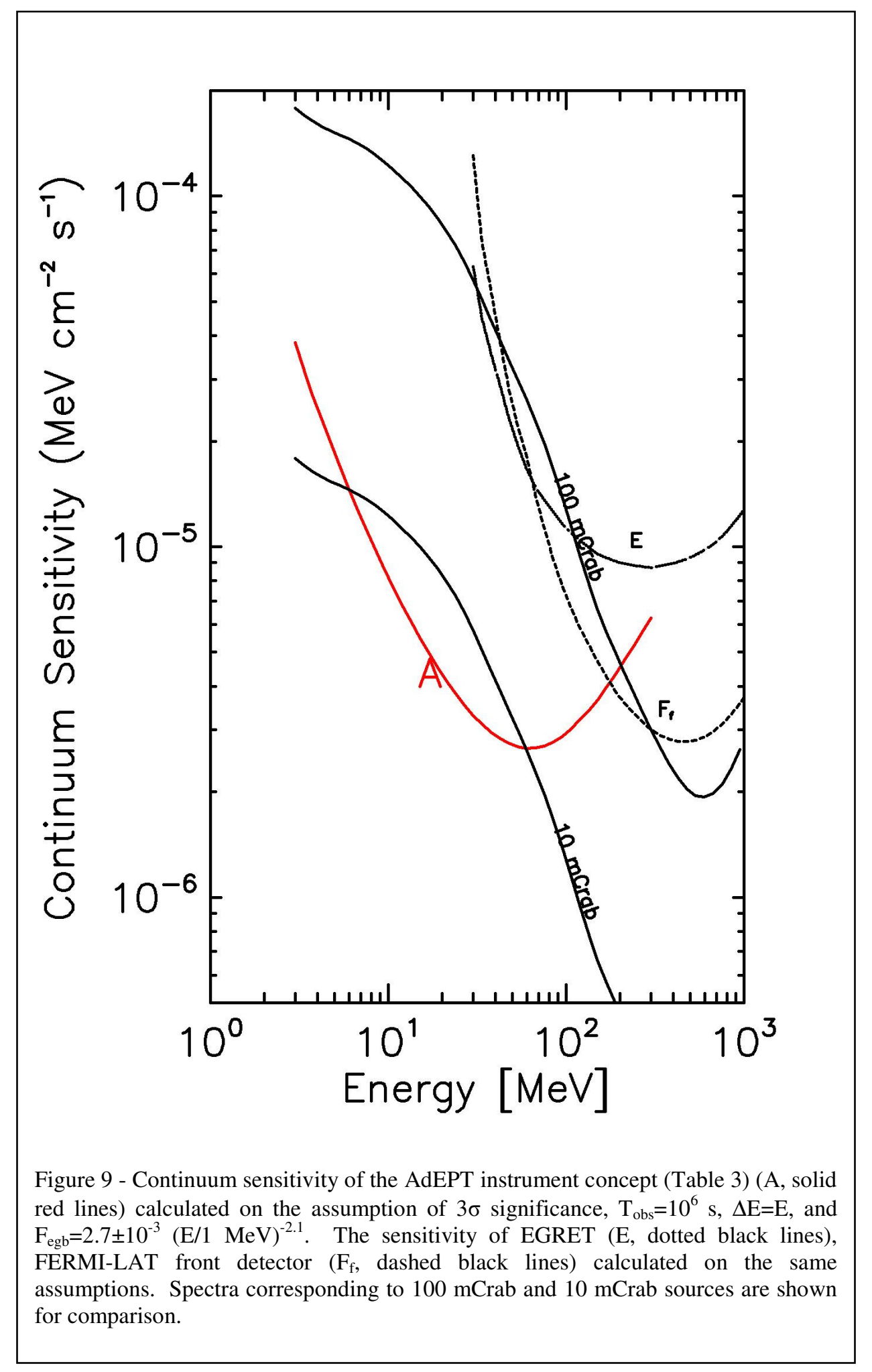




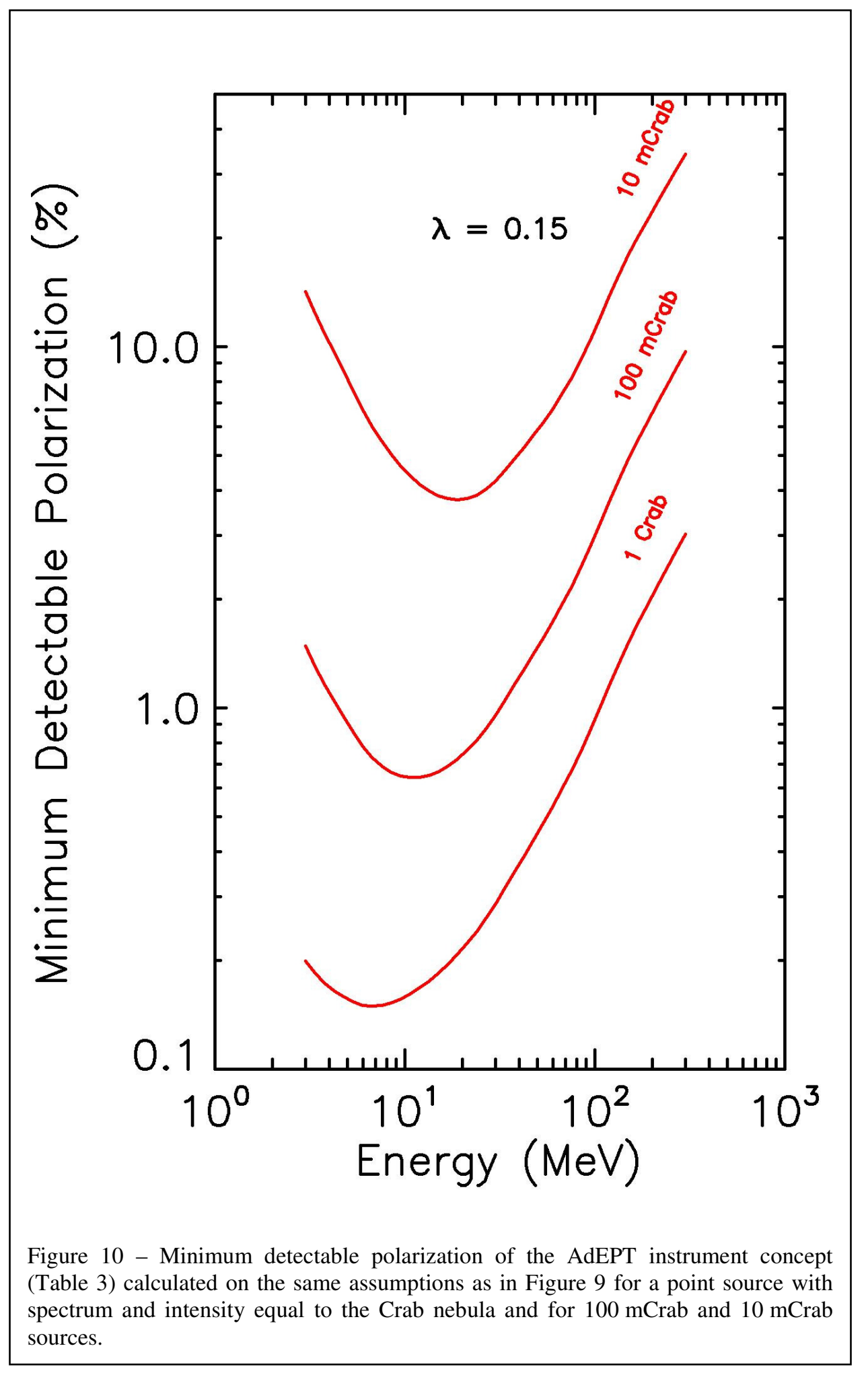




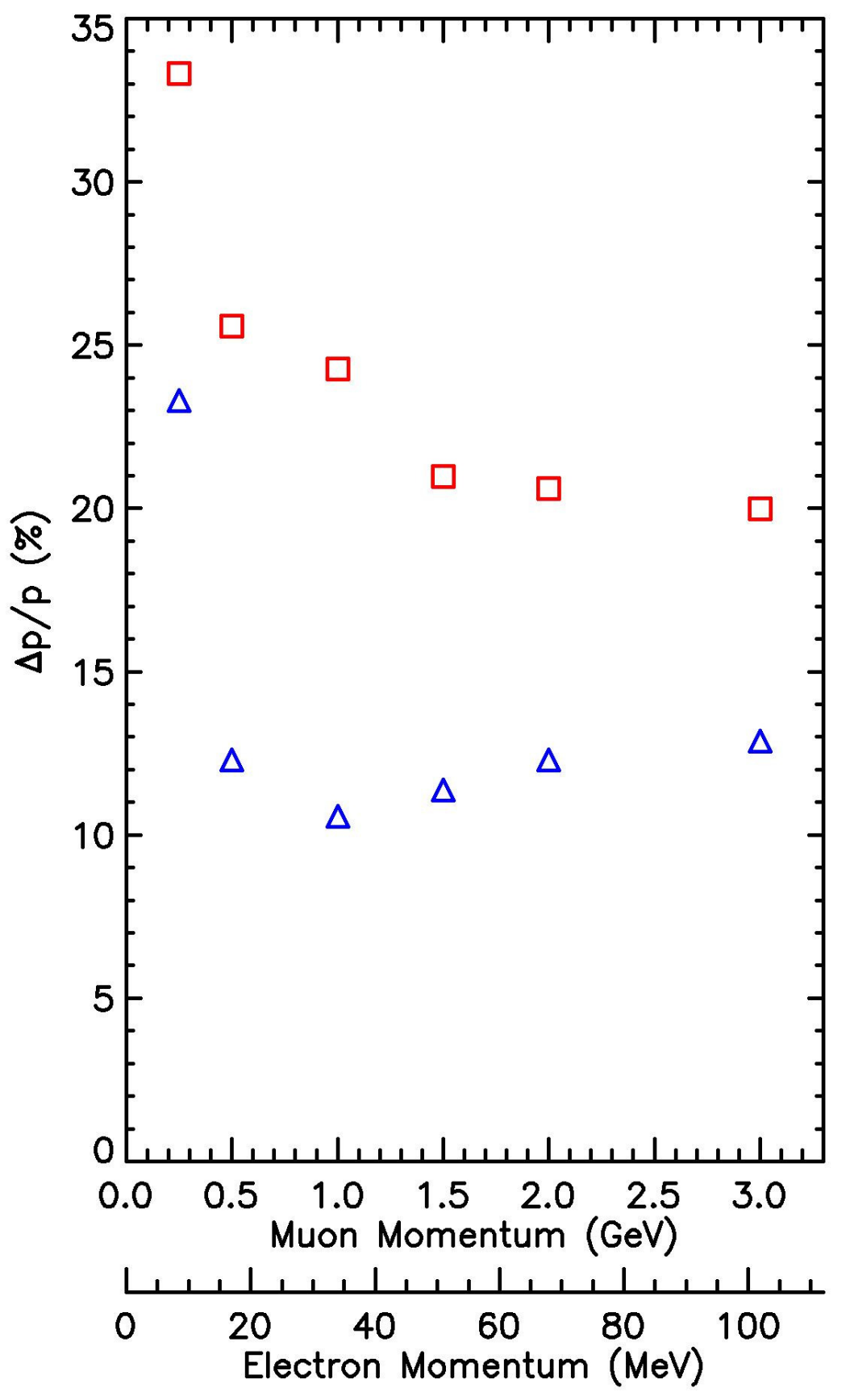

Figure 11 - Monte-Carlo simulation of the ICARUS momentum resolution using classical method (red squares) and the Kalman Filter method (blue triangles). The lower momentum axis has been scaled by the square root of the detector radiation length to give an estimated electron momentum resolution for AdEPT. Adapted from [89]. 